\title{
Estructura comunitaria de corales zooxantelados (Anthozoa: Scleractinia) en el arrecife coralino de Carrizales, Pacífico Mexicano
}

\author{
Héctor Reyes-Bonilla¹, Laura Elena Escobosa-González¹, Amílcar L. Cupul-Magaña², \\ Pedro Medina-Rosas ${ }^{2}$ \& Luis E. Calderón-Aguilera ${ }^{3 *}$ \\ 1. Universidad Autónoma de Baja California Sur. Departamento de Biología Marina. Apartado postal 19-B, CP 23080. \\ La Paz, B.C.S., México; hreyes@uabcs.mx \\ 2. Universidad de Guadalajara. Centro Universitario de la Costa. Ave. Universidad de Guadalajara \# 203, Delegación \\ Ixtapa. CP 48280, Puerto Vallarta, Jal., México; amilcar_cupul@yahoo.com.mx, pedromedinarosas@gmail.com \\ 3. Centro de Investigación Científica y de Educación Superior de Ensenada. Departamento de Ecología Marina. \\ Carretera Ensenada-Tijuana No. 3918, Zona Playitas, CP 22860, Ensenada, B. C., México; leca@cicese.mx \\ * Correspondencia
}

Recibido 19-IV-2012. Corregido 05-IX-2012. Aceptado 05-X-2012.

\begin{abstract}
Community structure of zooxanthellate corals (Anthozoa: Scleractinia) in Carrizales coral reef, Pacific coast, Mexico. Coral reefs in the Mexican Pacific and notably those of the continental coastline of Colima state are still poorly studied. Fortunately, recent efforts have been carried out by researchers from different Mexican institutions to fill up these information gaps. The aim of this study was to determine the ecological structure of the rich and undisturbed coral building communities of Carrizales by using the point transect interception method (25m-long). For this, three survey expeditions were conducted between June and October 2005 and September 2006; and for comparison purposes, the reef was subdivided according to its position in the bay, and depth ( 0 to $5 \mathrm{~m}$, and 6 to $10 \mathrm{~m}$ ). Thirteen coral species were observed in the area, with Pocillopora verrucosa as the most abundant, contributing up to $32.8 \%$ of total cover, followed by Porites panamensis and Pocillopora capitata with $11 \%$ and $7 \%$, respectively. Other species, Pocillopora damicornis, Pavona gigantea, Pocillopora eydouxi and Pocillopora inflata accounted for $1.5 \%$ to $2 \%$ of coral cover whereas the remaining five species had cover of less than $1 \%$. Seven of the observed species represented new records for Colima state coastline: Pocillopora eydouxi, P. inflata, P. meandrina, Pavona duerdeni, P. varians, Psammocora stellata and $P$. contigua. This last species is a relevant record, because it has never been observed before in the Eastern Pacific. Although there was no significant difference (ANOVA, $\mathrm{p}=0.478$ ) neither in the abundance between the sides of the bay, nor between the depths considered, and the shallow zone observed the higher coral cover. Live coral cover was up to $61 \%$, one of the highest ever reported for the Mexican Pacific, including the Gulf of California. The observed values of diversity $\left(H^{\prime}=0.44 \pm 0.02\right)$, uniformity $\left(J^{\prime}=0.76 \pm 0.02\right)$, and taxonomic distinctness index $\left(\Delta^{*}=45.87 \pm 3.16\right)$, showed that currently this is the most important coral reef of Colima coastline. Currently, this region does not show any disturbance effects, but the increasing economic development of Manzanillo, as one of the main commercial ports of Mexico, its proximity to the reef, and the burgeoning number of tourists, may have some ecosystem impacts, for which management and conservation plans for Colima coastline are highly recommended. Rev. Biol. Trop. 61 (2): 583-594. Epub 2013 June 01.
\end{abstract}

Key words: Colima, Eastern Pacific, diversity, taxonomic distinctness, new records, coral cover.

En décadas recientes se ha incrementado la información publicada sobre los corales arrecifales del Pacífico mexicano, y ya se cuenta con datos sobre su distribución geográfica y diversidad ecológica (Reyes-Bonilla et al. 2005, 2010), estructura comunitaria (Carriquiry
\& Reyes-Bonilla 1997, López-Pérez \& Hernández-Ballesteros 2004, López-Pérez et al. 2012), genética, reclutamiento y reproducción (Medina-Rosas et al. 2005, Saavedra-Sotelo et al. 2011, Rodríguez-Troncoso et al. 2011), e incluso sobre los genotipos de sus zooxantelas 
(Iglesias-Prieto et al. 2004, LaJeunesse et al. 2010) y su historia fósil (López-Pérez 2005, 2008). En general, puede decirse que la región presenta baja riqueza y abundancia coralina, y que es continuamente perturbada por tormentas tropicales y por el evento de Oscilación Sureña de El Niño (ENSO; Glynn \& LeyteMorales 1997, Carriquiry et al. 2001, ReyesBonilla et al., 2002). Las especies dominantes pertenecen al género Pocillopora (Lamarck 1818), y los arrecifes son pequeños, muy someros, y considerando su pequeño espesor, deben haberse establecido muy recientemente en el tiempo geológico (Reyes-Bonilla 2003, López-Pérez 2008).

A pesar de la información disponible, aún existen varias zonas de la costa occidental de México donde el conocimiento es incompleto y escaso, en especial el margen costero entre los $19^{\circ} \mathrm{N}$ y $17^{\circ} \mathrm{N}$ de latitud, que incluye los estados de Colima, Michoacán y Guerrero. Sobre los últimos dos sólo hay un par de trabajos publicados sobre las asociaciones coralinas (Brand 1958, Salcedo-Martínez et al. 1988), aunque Reyes-Bonilla (2003) y López-Perez et al. (2012) evidenciaron que en lugares como Acapulco y Zihuatanejo $\left(16^{\circ}-17^{\circ} \mathrm{N}\right)$ y El Faro de Bucerías $\left(18^{\circ} \mathrm{N}\right)$, la cantidad de coral, su espesor y la cementación de la estructura es tal, que se puede hablar de la existencia de verdaderos arrecifes. Además, existen referencias sobre estudios de físiología coralina y comunidades de peces en las localidades de La Boquita y Carrizales, en Colima $\left(19^{\circ} \mathrm{N}\right)$ (Chávez-Comparán \& Macías-Zamora 2006, Liñán-Cabello et al. 2006, 2010, Carignan et al. 2009), donde se mencionan formaciones coralinas importantes, pero no se presentan mayores datos. Con la finalidad de ampliar el conocimiento sobre los corales del Pacífico mexicano, el presente trabajo tuvo como objetivo describir la principal zona coralina del margen costero del estado de Colima, localizada en la pequeña bahía de Carrizales, a $14 \mathrm{~km}$ al oeste de Manzanillo, el principal puerto de altura del occidente de México.

\section{MATERIALES Y MÉTODOS}

Área de estudio: Desde la perspectiva oceanográfica, la costa del estado de Colima está relativamente bien caracterizada. La temperatura del agua superficial es alta $\left(27^{\circ} \mathrm{C}\right.$ a $28^{\circ} \mathrm{C}$ en promedio), ya que la costa recibe la influencia de la Corriente Occidental Mexicana, de corte tropical (Kessler 2006). La salinidad en época seca es de 35UPS, pero en temporada lluviosa (julio a octubre), puede bajar a 32UPS, mientras que los nutrientes inorgánicos (nitratos, silicatos y ortofosfatos) presentan concentraciones altas lejos de la costa, mientras que el amonio aumenta en sus cercanías (Olivos-Ortiz et al. 2006). La clorofila alcanza valores más altos en verano que en invierno, probablemente por enriquecimiento de origen pluvial, y además se registraron pequeños eventos de surgencia en temporadas de aguas frías, durante los meses de mayo y junio, los cuales también contribuyeron a aumentar la productividad oceánica local (Sosa-Avalos et al. 2006).

La Bahía de Carrizales $\left(19.09^{\circ} \mathrm{N}-104.44^{\circ}\right.$ W) tiene una extensión aproximada de 50 hectáreas, su boca está expuesta hacia el sureste y alcanza una profundidad máxima de $13 \mathrm{~m}$. El fondo está compuesto de arena en la zona central (más profunda), y hacia los márgenes someros este y oeste presenta crecimientos coralinos de considerable espesor $(>3 \mathrm{~m})$ y extensión (ca. 4ha), desarrollándose sobre roca granítica (Chávez-Comparán \& MacíasZamora 2006). La marea es semidiurna, con una amplitud promedio de $70 \mathrm{~cm}$ (Pacheco 1991). Chávez-Comparán \& Macías-Zamora (2006) reportaron que la comunidad de peces presenta 46 especies principales, donde el haemúlido Haemulon maculicauda (Gill 1862) y el pomacéntrido Stegastes acapulcoensis (Fowler 1944) dominan en abundancia.

Trabajo de campo y gabinete: Se realizaron tres expediciones (19-26 junio 2005, 20-27 octubre 2005 y 2-9 septiembre 2006) durante las cuales se condujeron censos de corales utilizando equipo de buceo autónomo. Se evaluó el porcentaje de cobertura coralina mediante el 
método de intersección de puntos, a lo largo de transectos de línea, de $25 \mathrm{~m}$ de largo, y en los que se registró el tipo de coral presente que intersectaba el transecto cada $50 \mathrm{~cm}(50$ puntos de muestreo por censo). Se corrieron 38 transectos, con los que se revisó $950 \mathrm{~m}$ lineales en total; 24 censos se situaron en el margen este de la bahía, y 14 en el oeste (debido a que su superficie es menor), con el fin de revisar las formaciones coralinas de la bahía en su totalidad. Las unidades de muestreo fueron distribuidas por nivel de profundidad, se efectuaron 19 en el nivel somero ( 0 a $6 \mathrm{~m})$, y 19 en el profundo (7 a 13m). Adicionalmente, durante la salida de 2006 se describió la zonación coralina con cinco transectos perpendiculares a la costa, cada uno de $100 \mathrm{~m}$ de largo, los cuales iniciaron desde la orilla $(0 \mathrm{~m})$ y recorrieron hasta la base del arrecife (-13m). Finalmente, se hicieron búsquedas de especies por medio de buceos errantes (buceando libremente fuera de los transectos), con el fin de completar el listado de los corales escleractinios presentes en la bahía. Las identificaciones de las especies se basaron en los trabajos de Cortés \& Guzmán (1998), Veron (2000) y Ketchum \& ReyesBonilla (2001).

Los datos obtenidos en el campo se procesaron para estimar varios indicadores de la estructura comunitaria coralina. Se calculó la cobertura total viva (porcentaje del fondo), la diversidad (índice de Shannon-Wiener, $\mathrm{H}^{\prime}$, base 10), la uniformidad (índice de Pielou, $\mathrm{J}^{\prime}$ ), y la diferenciación taxonómica ("taxonomic distinctness"; Clarke \& Warwick 2001b), un indicador que combina la abundancia, la riqueza y además el parentesco taxonómico de las especies encontradas. Todos estos índices fueron comparados mediante análisis de varianza de dos vías, con interacción, modelo II (Zar 2009), con una significancia del 95\%, y usando como factores de prueba la zona de estudio (este-oeste) y el nivel de profundidad (somero-profundo). Se aplicaron las pruebas $a$ priori de Bartlett (homoscedasticidad) y Kolmogorov-Smirnoff (normalidad), y a posteriori de Tukey (Zar 2009). Finalmente, se construyó una matriz de similitud entre transectos con el coeficiente de Bray-Curtis, y se hizo un análisis de escalamiento multidimensional no métrico (nMDS), con el fin de detectar patrones en la composición comunitaria. Este procedimiento genera un mapa de 3 dimensiones donde se sitúa cada transecto con base en su composición de especies y su abundancia, y estos tienden a agregarse en el gráfico con base en su similitud (Clarke \& Warwick 2001a).

\section{RESULTADOS}

Para la Bahía de Carrizales se documentó la presencia de 13 especies de corales hermatípicos en total, repartidos en cuatro géneros y cuatro familias (Cuadro 1). De ellas, solo dos no aparecieron en los transectos paralelos a la costa: Pocillopora meandrina (Dana 1846) y Psammocora contigua (Esper 1794), sino que se les encontró en los transectos perpendiculares, y en las revisiones del arrecife. El número total de especies para las zonas este y oeste fue el mismo (nueve) aunque la composición cambió; Pavona duerdeni (Vaughan 1907) y Porites lobata (Dana 1846) se observaron sólo en el oeste de la bahía y a profundidades mayores a 6m, mientras que Psammocora stellata (Verrill 1866) y Pavona varians (Verrill 1864) aparecieron exclusivamente en el lado este, ésta última a más de $6 \mathrm{~m}$ de profundidad (Cuadro 1 ). Sin embargo, considerando toda la información se reveló que las especies de coral se distribuyeron a todo lo largo de la bahía, aunque como se indicó antes, hubo diferencias en sus preferencias batimétricas y abundancias relativas.

Con respecto a la zonación, en Carrizales se distinguieron dos partes: la somera (del intermareal hasta $4-5 \mathrm{~m}$ ), que se caracterizó por tener representadas a todas las especies de Pocillopora, y con ocasionales apariciones de los demás géneros (Fig. 1). Ahí, el arrecife presentó un espesor aproximado de más de $3 \mathrm{~m}$, y su desarrollo era tal que había modificado totalmente el perfil del fondo en relación con el sustrato rocoso; además, la base arrecifal estaba claramente cementada. Por otro lado, la parte profunda $(>5 \mathrm{~m})$ se distinguió por la presencia de corales masivos (Porites y Pavona), 
CUADRO 1

Cobertura de corales arrecifales en Carrizales (promedio y error típico)

TABLE 1

Reef coral cover at Carrizales (average and standard error)

\begin{tabular}{lccccc}
\multicolumn{1}{c}{ Species } & \multicolumn{2}{c}{ East Zone } & \multicolumn{2}{c}{ West Zone } & Total cover (\%) \\
& Shallow & Deep & Shallow & Deep & \\
Pocillopora verrucosa $(\#)$ & $10.42 \pm 1.32$ & $9.63 \pm 2.54$ & $10.73 \pm 2.02$ & $2.01 \pm 3.79$ & $32.8 \pm 1.5$ \\
Pocillopora capitata & $5.62 \pm 1.83$ & $2.68 \pm 1.00$ & $2.63 \pm 1.17$ & $0.93 \pm 3.12$ & $11.9 \pm 0.9$ \\
Porites panamensis & $1.03 \pm 0.42$ & $3.89 \pm 1.06$ & $0.72 \pm 0.58$ & $1.50 \pm 2.27$ & $7.1 \pm 0.6$ \\
Pocillopora damicornis (\#) & $1.19 \pm 0.91$ & $0.46 \pm 0.37$ & $0.98 \pm 0.96$ & $0.10 \pm 0.40$ & $2.7 \pm 0.4$ \\
Pavona gigantea & $0.10 \pm 0.20$ & $1.45 \pm 0.53$ & $0.15 \pm 0.24$ & $0.88 \pm 2.23$ & $2.6 \pm 0.4$ \\
Pocillopora eydouxi (\#) & $0.67 \pm 0.42$ & $0.26 \pm 0.20$ & $0.15 \pm 0.33$ & - & $1.1 \pm 0.2$ \\
Pocillopora inflata & - & $0.36 \pm 0.37$ & $0.31 \pm 0.47$ & $0.31 \pm 0.97$ & $1.0 \pm 0.4$ \\
Pavona duerdeni $(\#)$ & - & $* * *$ & - & $0.62 \pm 2.40$ & $0.6 \pm 0.6$ \\
Psammocora stellata $(\#)$ & $0.05 \pm 0.10$ & $0.43 \pm 0.43$ & $* * *$ & $* * *$ & $0.5 \pm 0.1$ \\
Pavona varians(\#) & - & $0.41 \pm 0.57$ & - & $* * *$ & $0.4 \pm 0.2$ \\
Porites lobata (\#) & - & $* * *$ & - & $0.26 \pm 1.00$ & $0.3 \pm 0.3$ \\
\hline
\end{tabular}

(*) Especie presente en la zona pero no encontrada en los transectos. / (*) Species present in the area, but not found inside belt transects.

(\#) Especies inmigrantes del Pacífico Central y Occidental, sensu Veron (2000). / (\#) Immigrant species from the central and western Pacific, sensu Veron (2000).

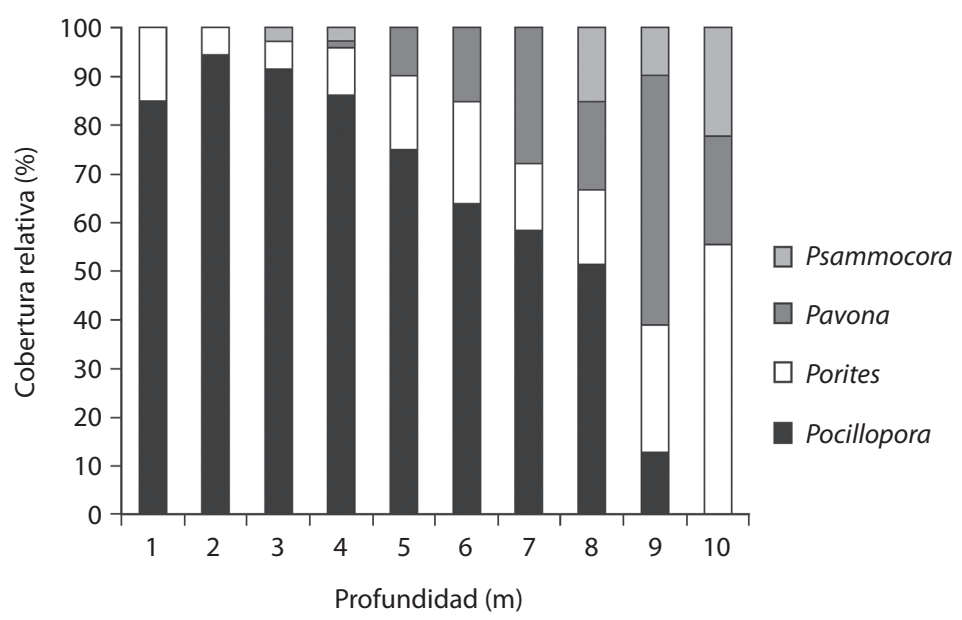

Fig. 1. Cobertura de coral por género en el gradiente de profundidad.

Fig. 1. Coral cover by genus along the depth gradient.

así como las dos especies de Psammocora, que aunque también se registraron en aguas someras, fueron menos comunes ahí. Esta sección presentaba porciones donde se podía observar el basamento rocoso arrecifal, peñascos grandes ( $>1 \mathrm{~m}$ de diámetro) y colonias aisladas y separadas del cuerpo principal del arrecife; llamaban la atención en particular los coralla de Pavona gigantea (Verrill 1869) y P. duerdeni, que podían alcanzar más de $1.5 \mathrm{~m}$ de altura y $3 \mathrm{~m}$ de diámetro. Finalmente, las únicas especies con una distribución vertical que abarcó 
ambas zonas fueron Pocillopora verrucosa (Ellis \& Solander 1786), Pocillopora capitata (Verrill 1864) y Porites panamensis (Verrill 1866) (Cuadro 1).

La cobertura total de coral vivo registrada en este estudio fue de 61.0 promedio $\pm 2.9 \%$ error típico; $\mathrm{N}=38$. El análisis de varianza no mostró diferencias significativas en cuanto a las zonas este y oeste $\left(\mathrm{F}_{1,34}=0.512, \mathrm{p}=0.478\right)$ aunque al tomar la profundidad como el factor, sí se presentaron diferencias estadísticas $\left(\mathrm{F}_{1,34}=9.610, \mathrm{p}=0.004\right)$, pues la parte somera presentó mayor cobertura de coral (Fig. 2). No hubo efecto de interacción $\left(\mathrm{F}_{1,34}=0.131\right.$, $\mathrm{p}=0.720$ ), aunque el promedio más alto se registró en el sitio este-somero $(72.55 \pm 0.03 \%)$ y el más bajo en oeste-profundo $(50.20 \pm 0.06 \%)$. El valor promedio de riqueza por transecto en el área de estudio fue de $4.05 \pm 0.18$ especies, y fue ligeramente mayor en la zona este $(4.25 \pm 0.23)$ y en los sitios más someros (4.10 \pm 0.25 ; Fig. 3 ). Sin embargo, los análisis estadísticos no mostraron diferencias significativas entre las zonas $\left(\mathrm{F}_{1,34}=2.305, \mathrm{p}=0.138\right)$, ni entre las dos profundidades $\left(\mathrm{F}_{1,34}=0.376, \mathrm{p}=0.544\right)$, o efectos de interacción ( $\left.\mathrm{F}_{1,34}=0.010, \mathrm{p}=0.920\right)$.
Con respecto a la abundancia por especies, se tuvo que $P$. verrucosa aportó el $32.8 \% \pm 1.45 \%$ a la cobertura absoluta (Cuadro $1)$, mientras $P$. panamensis y $P$. capitata obtuvieron entre el $11 \%$ y $7 \%$ en promedio; en conjunto estas tres especies contribuyeron con el $51.8 \%$ de la cobertura total de coral vivo de la zona de estudio, lo que equivalió a mas de tres cuartas partes de la abundancia coralina local. Las que siguieron en abundancia fueron $P$. damicornis, $P$. gigantea, Pocillopora eydouxi (Milne Edwards \& Haime 1860) y Pocillopora inflata (Glynn 1999) (1.5\% a 2\% del fondo), y las cuatro restantes tuvieron valores absolutos muy bajos, menores al 1\% (Cuadro 1).

El arrecife de Carrizales presentó un valor promedio de diversidad de $0.44 \pm 0.02$ decits/ ind. En la zona este, $H^{\prime}$ fue significativamente más alto que en la oeste $\left(\mathrm{F}_{1,36}=4.478\right.$, $\mathrm{p}=0.042$, Fig. 3), mientras que en relación a la profundidad no hubo diferencias significativas $\left(\mathrm{F}_{1,34}=0.996, \mathrm{p}=0.325\right)$. Sin embargo, se observó que entre los 7 y $13 \mathrm{~m}$ (zona profunda), la diversidad fue ligeramente mayor a la de la parte somera. Al comparar las zonas y las profundidades en conjunto, el análisis de
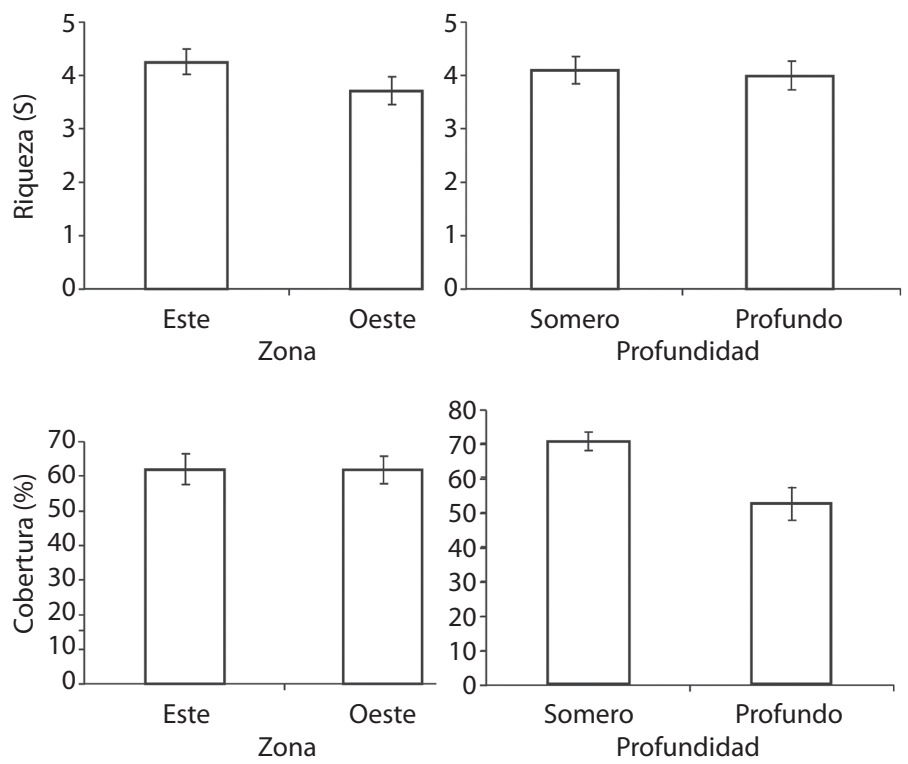

Fig. 2. Cobertura y riqueza de coral por zona y profundidad. Fig. 2. Coral cover and species richness by zone and depth. 

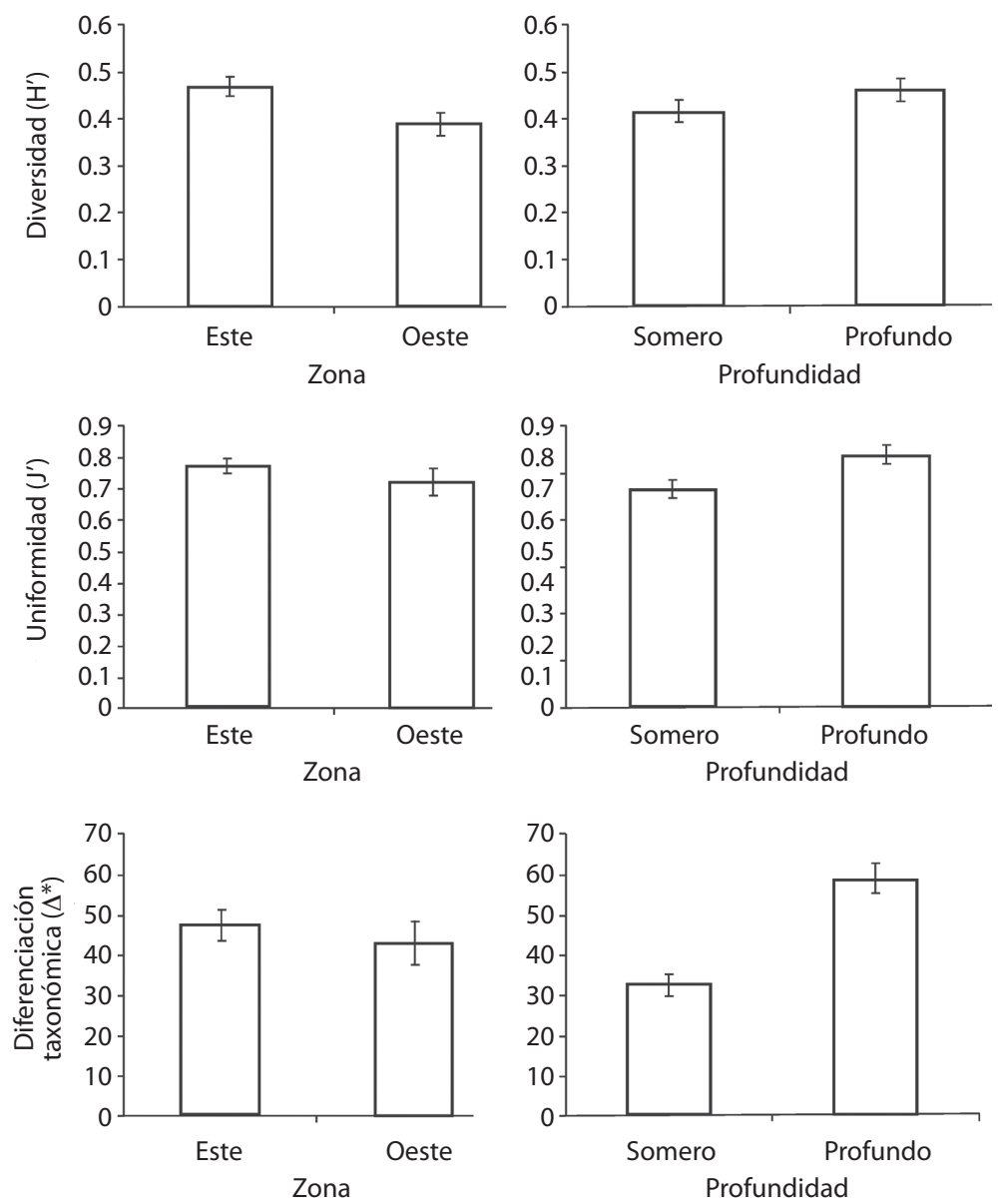

Fig. 3. Diversidad, equidad y diferenciación taxonómica por zona y profundidad.

Fig. 3. Diversity, evenness and taxonomic distinctness by zone and depth.

varianza no demostró interacción $\left(\mathrm{F}_{1,34}=0.061\right.$, $\mathrm{p}=0.807$ ) aunque en la figura 3 se puede observar que el sitio con mayor valor de $\mathrm{H}^{\prime}$ fue el este-profundo $(0.48 \pm 0.03)$.

La uniformidad promedio del área fue de $0.76 \pm 0.02$. Se registró un valor ligeramente mayor en la zona este que en el oeste, pero no hubo diferencias significativas entre ambas $\left(F_{1,34}=0.006, p=0.394\right)$. En cambio, la uniformidad entre las dos profundidades sí difirió $\left(\mathrm{F}_{1,34}=5.704, \mathrm{p}=0.023\right)$, siendo mayor en sitios profundos que someros (Fig. 3). No se detectaron efectos de interacción sitio-profundidad $\left(\mathrm{F}_{1,34}=0.086, \mathrm{p}=0.772\right)$ aunque observamos mayor uniformidad en los transectos del lado este-profundo $(0.81 \pm 0.03)$ y menos en el oestesomero $(0.68 \pm 0.05)$. Finalmente, los valores promedios de diferenciación taxonómica en la zona este y oeste no presentaron diferencias significativas $\left(\mathrm{F}_{1,34}=0.453, \mathrm{p}=0.505\right.$; Fig. 3$)$. No obstante, al cambiar el factor por la profundidad, el análisis de varianza mostró discrepancias muy claras $\left(\mathrm{F}_{1,34}=31.699, \mathrm{p}<0.0001\right)$ pues las zonas profundas fueron mucho más diversas bajo esta perspectiva. El análisis de varianzas de dos vías para las zonas y profundidades no mostró efectos de interacción $\left(\mathrm{F}_{1,34}=0.075, \mathrm{p}=0.786\right)$. 
El escalamiento multidimensional no métrico tuvo un stress de 0.14 (Fig. 4) y el gráfico muestra la gran dispersión de los puntos que representan el este y oeste de la Bahía de Carrizales; eso indica que la composición de corales hermatípicos es semejante en la zona de estudio. A pesar de ello, al observar los datos en función a la profundidad (Fig. 4), los censos efectuados en aguas someras se encuentran más agrupados que los profundos (al lado izquierdo del gráfico), indicando que existe comparativamente una mayor afinidad en su composición coralina.

\section{DISCUSIÓN}

La Bahía de Carrizales es un sitio pequeño, y el arrecife aproximadamente mide apenas cuatro hectáreas, sin embargo durante el trabajo de campo se observaron 13 especies de coral, lo que representa el 39\% del total de escleractinios arrecifales conocidos en México (Reyes-Bonilla 2003, Reyes-Bonilla et al. 2005). De las 13 indicadas, siete no habían sido registradas para el margen continental del estado de Colima (Pocillopora eydouxi, P. inflata, P. meandrina, Pavona duerdeni, $P$. varians, Psammocora stellata y P. contigua (Reyes-Bonilla et al. 2005) y con estos nuevos registros se eleva la cifra para la región hasta 15 especies, un valor alto comparado a otras zonas de la costa del occidente de México (Reyes-Bonilla et al., 2010). Aquí se sugiere que la aceptable riqueza de especies coralinas puede deberse a que según Glynn et al. (1996), Reyes-Bonilla et al. (1999) y Perez-Vivar et al. (2006), la costa de México
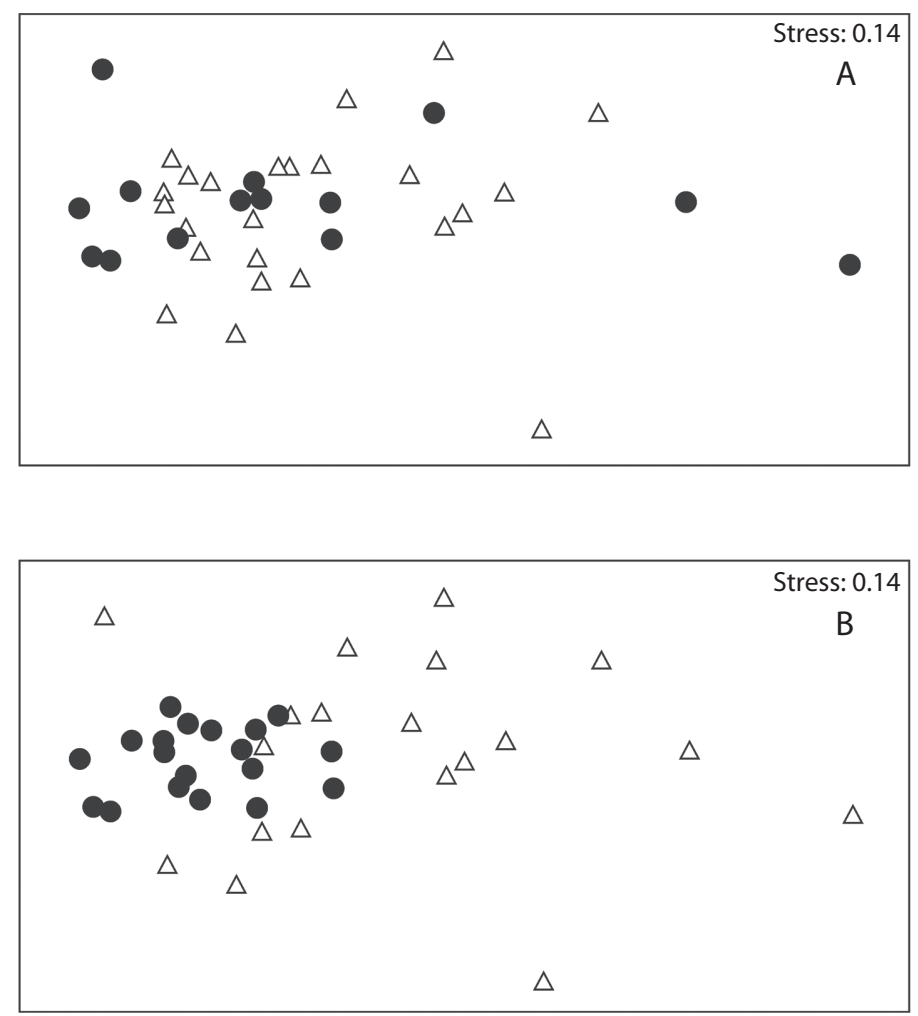

Fig. 4. Escalamiento multidimensional no métrico, por zona y profundidad. a) triángulos: zona este, círculos: zona oeste. b) círculos: arrecife somero; triángulos: arrecife profundo.

Fig. 4. Non metric multidimensional scaling, by zone and depth. a) triangles: east zone, circles: west zone. b) circles: shallow reef; triangles: deep reef. 
entre los $18^{\circ} \mathrm{N}$ y $20^{\circ} \mathrm{N}$ representa la zona principal de acopio de larvas inmigrantes desde el Pacífico central; esta observación sirve para explicar por qué la fauna coralina en Carrizales es fundamentalmente inmigrante del oeste (62\% de las especies).

De los siete nuevos registros llama particularmente la atención la ocurrencia de Psammocora contigua, un coral oriundo del Indo Pacífico y que no había sido visto en el Pacífico Oriental con anterioridad (Reyes-Bonilla 2002). Sería de esperarse que si la especie ha llegado a Colima por colonización sucesiva de sitios adyacentes ("stepping stones"), entonces esté presente en sitios como las Islas Revillagigedo o el Atolón Clipperton, pero eso no ocurre (Glynn et al. 1996, Carricart-Ganivet \& Reyes-Bonilla 1999, Ketchum \& Reyes-Bonilla 2001). Consecuentemente, aquí se propone la hipótesis que las larvas pudieron haber arribado en agua de lastre en alguno de los cientos de barcos que cruzan el Pacífico y descargan productos en Manzanillo, lo que constituiría el primer ejemplo de una especie invasora de coral en el oeste de México. Es importante realizar un análisis temporal de la población en Carrizales para confirmar su establecimiento en el país, o si su presencia fue transitoria, como la de Acropora valida (Dana 1846) y de Porites rus (Forskål 1775) en América Central luego del evento de El Niño de 1982-1983 (Guzmán \& Cortés 1993).

Además de su riqueza, el arrecife de Carrizales llamó la atención por su notablemente alta cobertura coralina $(61.0+2.9 \%)$, la cual es superior a la encontrada en cualquier otro sitio del Pacífico mexicano registrado en la literatura y que haya sido revisado posteriormente al evento de mortalidad coralina masiva causado por el ENSO de 1997-1998 (Carriquiry et al. 2001, Reyes-Bonilla 2001, López-Pérez \& Hernández-Ballesteros 2004). Una de las causas probables de esta alta cobertura local puede ser el efecto de fragmentación de ramas de Pocillopora spp., que favorece su dispersión local dentro de arrecifes en todo el Pacífico Oriental (Glynn \& Ault 2000). Además, el sitio es somero y recibe alta iluminación
(Olivos-Ortiz et al. 2006), lo que propicia el desarrollo coralino, y finalmente, la bahía está poco perturbada por actividades humanas ya que solo se puede acceder por mar (ChávezComparán \& Macías-Zamora 2006), aunque las actividades de buceo libre y autónomo van a la alza. Se debe llamar la atención de las autoridades para que controlen el flujo de personas en aras de la conservación del parche coralino más importante del estado.

No hay información sobre la respuesta de los corales en la zona costera de Colima a la elevación de temperatura causada por los eventos ENSO de 1987-1988 y 1997-1998, aunque (Reyes-Bonilla 2003) indicó que en las Islas Revillagigedo el blanqueamiento de coral había sido muy poco en 1997, y Filonov et al. (2000) y Filonov \& Novotryasov (2007) mostraron que lo angosto de la plataforma continental en el estado de Colima permite una eficiente mezcla de aguas profundas y someras, e impide elevaciones drásticas de la temperatura superficial. A pesar de lo anterior, Liñán-Cabello et al. (2006) indicaron ligeras pérdidas de coloración en colonias de Pocillopora de La Boquita (situada a unos $10 \mathrm{~km}$ de Carrizales) en 2004, pero sin daños mayores a las poblaciones o a la comunidad en general. Este problema pudo ser producto de que de agosto a octubre de ese año la temperatura pasó $\operatorname{los} 30^{\circ} \mathrm{C}$, aunque no se excedió el umbral de blanqueamiento de $30.6^{\circ} \mathrm{C}$ (equivalente a la temperatura de $1{ }^{\circ} \mathrm{C}$ sobre el promedio del mes más cálido en los años 1984-2008; Carriquiry et al. 2001). La relativa estabilidad de este factor también debe tener influencia en la alta riqueza y abundancia coralina en Carrizales, y ello lleva a sugerir que el buen estado del arrecife parece resultar de la ausencia de perturbaciones ambientales crónicas o intensas, y el poco uso humano.

La zona de estudio mostró la zonación típica de los arrecifes del Pacífico Oriental (Cortés \& Jiménez 2003), con el género Pocillopora como abundante en aguas someras, Pavona y Porites en aguas profundas, y Psammocora como miembro secundario. La especie dominante en Carrizales fue $P$. verrucosa, coincidente con lo que ocurre en el Golfo de 
California (Reyes-Bonilla 2003), pero marcando una diferencia del patrón observado en Nayarit, Guerrero y Oaxaca, donde P. damicornis forma la mayor parte de los arrecifes (Carriquiry \& Reyes-Bonilla 1997, López Pérez \& Ballesteros 2004, Perez-Vivar et al. 2006). La predominancia de los corales pocilloporidos en el Pacífico Oriental se debe fundamentalmente a su velocidad de crecimiento (Guzmán \& Cortés 1993) y su citada habilidad para crecer y ocupar espacio por fragmentación, ya que repetidos estudios han mostrado que en el Pacífico mexicano el reclutamiento sexual de este género es limitado (Medina-Rosas et al. 2005, López-Pérez \& Leyte-Morales 2007, Chávez-Romo et al. 2008, Rodríguez-Troncoso et al. 2011).

Los análisis estadísticos indicaron que no hubo diferencias significativas en ningún indicador comunitario entre la parte este y oeste de Carrizales, y el NMDS mostró la semejanza en composición a todo lo largo de la bahía; así, se puede afirmar que en general el arrecife funciona como una unidad en el marco espacial. La única excepción al patrón fue $\mathrm{H}^{\prime}$, para la cual el margen oriental de la bahía tuvo mayores valores. Esto puede explicarse por el hecho que en la porción profunda del lado este tuvo más alta riqueza total (nueve especies en los transectos), y presentó mayor cantidad de microambientes (rocas grandes, cuevas, parches arenosos), lo que favorece la variedad de especies y su mayor semejanza en abundancia relativa, factores ambos que aumentan la diversidad (Clarke \& Warwick 2001b).

Sin embargo, la situación difiere notablemente en relación con la profundidad, ya que hubo más cobertura de coral en aguas someras, y mayor uniformidad y diferenciación taxonómica en las profundas. Los tres resultados se pueden interpretar en conjunto de la siguiente forma: la alta cobertura en partes someras se debe a la marcada dominancia de Pocillopora spp., que homogeniza la composición específica, pero a su vez disminuye la presencia y abundancia relativa de los demás géneros y consecuentemente deprime $\mathrm{J}^{\prime} \mathrm{y} \Delta^{*}$. En aguas profundas Pocillopora spp. pierde su dominancia al bajar la intensidad lumínica y tener dificultades metabólicas (Iglesias-Prieto et al. 2004), y ello da lugar a un mayor equilibrio en las abundancias de las especies y los grupos mayores presentes (géneros, familias). Por otra parte, Brown et al. (2002) encontraron que los valores de diferenciación taxonómica tendieron a ser bajos en aguas someras de arrecifes de Tailandia, ya que la desecación y la alta intensidad de luz solo permite la permanencia de ciertos géneros; en zonas más profundas, el stress físico disminuye y ello permite aumentar la variedad de táxones de niveles mayores; todo esto también parece coincidir con lo observado en Carrizales.

Acerca de las diferencias en la cobertura coralina, el hecho que sea mayor en sitios someros es un fenómeno que se presenta repetidamente en arrecifes de México (Carriquiry \& Reyes-Bonilla 1997, Glynn \& Leyte-Morales 1997) y en América Central (Glynn \& Ault 2000, Cortés 2003). La explicación que se ha ofrecido sobre las diferencias batimétricas es que procesos físicos como el oleaje, la sedimentación y principalmente la cantidad de luz, pueden favorecer la abundancia de Pocillopora spp. y con ello la mayor cobertura de coral cerca de la superficie (McCulloch et al. 2003, Iglesias-Prieto et al. 2004). Por otro lado, la abundancia disminuye en aguas más profundas ya que ellas están dominadas por especies de crecimiento lento con menor capacidad de fragmentación (Reyes-Bonilla \& Calderón-Aguilera 1994, Reyes-Bonilla et al. 1999). La diferencia en cobertura se ve acrecentada por la mayor intensidad de depredación a las colonias al aumentar el número de peces coralívoros (Guzmán \& Cortés 1993, Reyes-Bonilla \& Calderon-Aguilera 1999), y porque Pavona y Porites parecen tener adaptaciones a la baja intensidad lumínica y ello hace que sean más comunes en zonas cercanas a la base arrecifal (Iglesias-Prieto et al. 2004, LaJeunesse et al. 2010).

Si se compara a escala regional, los valores de $\mathrm{H}^{\prime}$ y J' en Carrizales son similares a los reportados para Cabo Pulmo $\left(23^{\circ} \mathrm{N}\right)$, Careyeros $\left(20^{\circ} \mathrm{N}\right)$ y Huatulco $\left(15^{\circ} \mathrm{N}\right)$ (Carriquiry \& 
Reyes-Bonilla 1997, Reyes-Bonilla \& Calderon-Aguilera 1999, López-Pérez \& HernándezBallesteros 2004), aunque ligeramente más bajos. Esta observación implica que la dominancia de los corales pocilopóridos en Carrizales parece ser mayor a lo normal, y quizá eso se deba a que como se mencionó antes, la región de Manzanillo sufre relativamente pocas perturbaciones anuales. Además de la homgeneidad interanual en la temperatura, la zona recibió el embate de solo cinco huracanes entre 1980 y 2006 (www.nhc.noaa.gov). De acuerdo con Connell et al. (1997) y Karlson (1999) sitios que sufren muy pocos o demasiados disturbios presentan baja diversidad en relación con los que sufren el llamado "disturbio intermedio", esto originado por un lado por la exclusión competitiva y por otro por la dificultad de recuperación en caso de desastres.

En conclusión, la Bahía de Carrizales, Colima cuenta con un arrecife bien desarrollado, caracterizado por alta riqueza y abundancia coralina en comparación con otras zonas del Pacífico mexicano, aunque su diversidad es ligeramente baja dada la gran dominancia de especies del género Pocillopora. La calidad del sitio se explica por la ausencia de perturbaciones naturales crónicas o intensas, y por el reducido nivel de uso humano que ha tenido. Considerando el importante desarrollo portuario que se está llevando a cabo en la región de Manzanillo (uno de los principales puertos comerciales de México y situado a unos cuantos kilómetros de Carrizales) y a la cada vez mayor afluencia turística a la zona, sugerimos que debido a la relevancia estatal de este arrecife, deben establecerse estrategias de manejo adecuadas para mantener su buen estado de conservación.

\section{AGRADECIMIENTOS}

Los fondos para realizar la investigación fueron proporcionados por la Comisión Nacional para el Conocimiento y Uso de la Biodiversidad (proyecto AS007, a LECA).

\section{RESUMEN}

El conocimiento ecológico de corales arrecifales en el Pacífico mexicano es escaso, por lo que el objetivo de este trabajo fue determinar la estructura de la comunidad de corales hermatípicos en el arrecife de Carrizales, Colima, mediante el uso de transectos y buceo autónomo (junio y octubre 2005, septiembre 2006). De las 13 especies de corales encontradas, Pocillopora verrucosa fue la más abundante y siete representan nuevos registros, sobresaliendo Psammocora contigua, primer registro para el Pacífico Oriental. No hubo diferencias significativas de abundancia entre profundidades, pero la zona somera presenta una mayor cobertura. Este sitio presenta una de las riquezas y cobertura de coral más alta $(61 \%)$ en el Pacífico Mexicano y valores de diversidad $\left(H^{\prime}=0.44 \pm 0.02\right)$, uniformidad $\left(J^{\prime}=0.76 \pm 0.02\right)$, y de diferenciación taxonómica $\left(\Delta^{*}=45.87 \pm 3.16\right)$ relativamente altos. Actualmente la región no presenta grandes perturbaciones pero el creciente desarrollo económico de Manzanillo, uno de los principales puertos comerciales del país, además del creciente número de turistas, podrían afectar al arrecife, por lo que se sugiere implementar medidas de protección con el fin de mantener al arrecife más importante del litoral de Colima.

Palabras clave: Colima, Pacífico Oriental, diversidad, diferenciación taxonómica, nuevos registros, cobertura coralina.

\section{REFERENCIAS}

Brown, B.B., K.C. Clarke \& R.W. Warwick. 2002. Serial patterns of biodiversity change in corals across shallow reef flats in Ko Phuket, Thailand, due to the effects of local (sedimentation) and regional (climatic) perturbations. Mar. Biol. 141: 21-29.

Brand, D.B. 1958. Coastal study of western Mexico, part 2. University of Texas, Austin, EEUU.

Carignan, M.O., K.H.M. Cardozo, D. Oliveira-Silva, P. Colepicolo \& J.I. Carreto. 2009. Palythine-threonine, a major novel mycosporine-like amino acid (MAA) isolated from the hermatypic coral Pocillopora capitata. J. Photoch. Photobio. B 94: 191-200.

Carricart-Ganivet, J.P. \& H. Reyes-Bonilla. 1999. New and previous records of Scleractinian corals from Clipperton Atoll, Eastern Pacific. Pac. Sci. 53: 370-375.

Carriquiry, J.D. \& H.R. Bonilla. 1997. Estructura de la comunidad y distribución geográfica de los arrecifes coralinos de Nayarit, Pacífico de México. Cienc. Mar. 23: 227-248.

Carriquiry, J.D., A. Cupul-Magaña, F.A. Rodríguez-Zaragoza \& P. Medina-Rosas. 2001. Coral bleaching and mortality in the Mexican Pacific during the $199798 \mathrm{El}$ Nino and prediction from a remote sensing approach. Bull. Mar. Sci. 69: 237-249. 
Chávez Comparán, J.C. \& R. Macías-Zamora. 2006. Structure of Reef Fish Communities in the Littoral of Colima, Mexico. J. Biol. Sci. 6: 65-75.

Chavez-Romo, H. \& H. Reyes-Bonilla. 2007. Sexual reproduction of the coral Pocillopora damicornis in the southern Gulf of California, Mexico. Cienc. Mar. 33: 495-501.

Chávez-Romo, H., F. Correa-Sandoval, D. Paz-Garcl̀a, H. Reyes-Bonilla, R. López-Pérez, P. Medina-Rosas \& M. Hernández-Cortés. 2008. Genetic structure of the scleractinian coral, Pocillopora damicornis, from the Mexican Pacific, p. 429-433. In B. Riegl (ed.). Proceedings of the 11th International Coral Reef Symposium, Ft. Lauderdale, Florida, EEUU.

Clarke, K.R. \& R.M. Warwick. 2001a. Change in marine communities. An approach to statistical analisys and interpretation. Primer-E, Londres, Reino Unido.

Clarke, K.R. \& R.M. Warwick. 2001b. A further biodiversity index applicable to species lists: variation in taxonomic distinctness. Mar. Ecol. Prog. Ser. 216: 265-278.

Connell, J.H., T.P. Hughes \& C.C. Wallace. 1997. A 30 -year study of coral abundance, recruitment, and disturbance at several scales in space and time. Ecol. Monogr. 67: 461-488

Cortés, J. 2003. Coral Reefs of the Americas: An introduction to Latin American Coral Reefs, p. 1-7. In J. Cortés (ed.). Latin American Coral Reefs. Elsevier, Amsterdam.

Cortés, J. \& H. Guzmán. 1998. Organismos de los arrecifes coralinos de Costa Rica: descripción, distribución geográfica e historia natural de los corales zooxantelados (Anthozoa: Scleractinia) del Pacífico. Rev. Biol. Trop. 46: 55-92.

Cortés, J. \& C. Jiménez 2003. Corals and Coral Reefs of the Pacific of Costa Rica: history, research and status, p. 361-385 In J. Cortés (ed.). Latin American Coral Reefs. Elsevier, Amsterdam.

Filonov, A. \& V. Novotryasov. 2007. On a spectrum of nonlinear internal waves in the oceanic coastal zone. Non. Linear Proc. Geoph. 14: 757-762.

Filonov, A., I.E. Tereshchenko, C.O. Monzón, M.E. González-Ruelas \& E. Godínez-Domínguez. 2000. Variabilidad estacional de los campos de temperatura y salinidad en la zona costera de los estados de Jalisco y Colima, México. Cienc. Mar. 26: 303-321.

Glynn, P.W. \& J.S. Ault. 2000. A biogeographic analysis and review of the far eastern Pacific coral reef region. Coral Reefs 19: 1-23.

Glynn, P.W. \& G.E. Leyte-Morales. 1997. Coral reefs of Huatulco, West México: reef development in upwelling Gulf of Tehuantepec. Rev. Biol. Trop. 45: 1033-1047.
Glynn, P.W., J.E.N. Veron \& G.M. Wellington. 1996. Clipperton Atoll (eastern Pacific): oceanography, geomorphology, reef-building coral ecology and biogeography. Coral Reefs 15: 71-99.

Guzmán, H.M. \& J. Cortés. 1993. Arrecies coralinos del Pacífico Oriental Tropical: Revisión y perspectivas. Rev. Biol. Trop. 41: 535-557.

Iglesias-Prieto, R., V.H. Beltran, T.C. LaJeunesse, H. Reyes-Bonilla \& P.E. Thome. 2004. Different algal symbionts explain the vertical distribution of dominant reef corals in the eastern Pacific. Pr. Roy. Soc. B-Biol Sci. 271: 1757-1763.

Karlson, R.H. 1999. Dynamics of Coral Communities. Kluwer, Norwell, Massachussetts EEUU.

Kessler, W.S. 2006. The circulation of the eastern tropical Pacific: A review. Prog. Ocean. 69: 181-217.

Ketchum, J.T. \& H. Reyes-Bonilla. 2001. Taxonomy and distribution of the hermatypic corals (Scleractinia) of the Revillagigedo Archipelago, Mexico. Rev. Biol. Trop. 49: 803-848.

LaJeunesse, T.C., R. Smith, M. Walther, J. Pinzón, D.T. Pettay, M. McGinley, M. Aschaffenburg, P. MedinaRosas, A.L. Cupul-Magaña, R.A. López-Pérez, H. Reyes-Bonilla \& M.E. Warner. 2010. Host-symbiont recombination versus natural selection in the response of coral-dinoflagellalate symbiosis to environmental disturbances. Pr. Roy. Soc. B-Biol Sci. 277: 2925-2934

Liñán-Cabello, M.A., L. Flores-Ramírez, J.S. ZacaríasSalinas, O. Hernández-Rovelo \& C. Lezama-Cervantes. 2006. Correlation of chlorophyll a and total carotenoid concentrations with coral bleaching from locations on the Pacific coast of Mexico. Mar. Freshw. Behav. Phy. 39: 279-291.

Liñán-Cabello, M.A., L.A. Flores-Ramírez, T. ZentenoSavin, N.O. Olguín-Monroy, R. Sosa-Avalos, M. Patiño-Barragan \& A. Olivos-Ortiz. 2010. Seasonal changes of antioxidant and oxidative parameters in the coral Pocillopora capitata on the Pacific coast of Mexico. Mar. Ecol. 31: 407-417.

López-Pérez, R.A. 2005. The Cenozoic hermatypic corals in the eastern Pacific: history of research. Earth-Sci. Rev. 72: 67-87.

López-Pérez, R.A. 2008. Fossil corals from the Gulf of California, México:Still a depauperate fauna but it bears more species than previously thought. Proc. Cal. Acad. Sci: 13: 515-531.

López-Pérez, R.A. \& L.M. Hernández-Ballesteros. 2004. Coral community structure and dynamics in the Huatulco Area, Western Mexico. Bull. Mar. Sci. 75: 453-472.

López Pérez, R.A., M. Mora-Pérez \& G.E. Leyte-Morales. 2007. Coral (Anthozoa: Scleractinia) Recruitment at Bahías de Huatulco,Western México: Implications 
for coral community structure and dynamics. Pac. Sci. 61: 355-369.

López-Pérez, R.A., L.E. Calderón-Aguilera, H. ReyesBonilla, J.D. Carriquiry, P. Medina-Rosas, A.L. Cupul-Magaña, M.D. Herrero-Pérezrul, H.A. Hernández-Ramírez, M.A. Ahumada-Sempoal \& B.M. Luna-Salguero. 2012. Coral commuities and reefs from Guerrero, southern Mexican Pacific. Mar. Ecol. 1: 1-12.

McCulloch, M., S. Fallon, T. Wyndham, E. Hendy, J. Lough \& D. Barnes. 2003. Coral record of increased sediment flux to the inner Great Barrier Reef since European settlement. Nature 421: 727-730.

Medina-Rosas, P., J.D. Carriquiry \& A.L. Cupul-Magana. 2005. Recruitment of Porites (Scleractinia) on artificial substrate in reefs affected by the 1997-98 El Nino in Banderas Bay, Mexican Pacific. Cienc. Mar. 31: 103-109.

Olivos-Ortiz, A., J.J. Salas-Pérez, G. García-Uribe, M.A. Galicia-Pérez, E. Torres-Orozco \& S.I. QuijanoScheggia. 2006. Distribución horizontal de nutrientes y clorofila- $a$ sobre la plataforma continental del estado de Colima durante 2002, p. 53-65 In M.C. Jiménez-Quiroz \& E. Espino-Barr (ed.). Los recursos pesqueros y acuícolas de Jalisco, Colima y Michoacán. Instituto Nacional de la Pesca, México.

Pacheco, S.P. 1991. Pacífico Tropical Mexicano. Cabo Corrientes a la frontera con Guatemala, p. 151-175. In G. de la Lanza Espino (ed.). Oceanografía de mares mexicanos, México.

Perez-Vivar, T.L., H. Reyes-Bonilla \& C. Padilla. 2006. Corales pétreos (Scleractinia) de las Islas Marías, Pacífico de México. Cienc. Mar. 32: 259-270.

Reyes-Bonilla, H. 2001. Effects of the 1997-1998 El Niño Southern Oscillation on coral communities of the Gulf of California, Mexico. Bull. Mar. Sci. 69: 251-266.

Reyes-Bonilla, H. 2002. Checklist of valid names and synonyms of stony corals (Anthozoa: Scleractinia) from the eastern Pacific. J. Nat. Hist. 36: 1-13.

Reyes-Bonilla, H. 2003. Coral reefs of the Pacific coast of México, p. 331-349 In J. Cortés (ed.). Latin American Coral Reefs. Elsevier, Amsterdam.

Reyes Bonilla, H. \& L. Calderón-Aguilera. 1994. Parámetros problacionales de Porites panamensis (Anthozoa: Scleractinia), en el arrecife de Cabo Pulmo, México. Rev. Biol. Trop. 42: 121-128.

Reyes Bonilla, H., T.L. Pérez Vivar \& J.T. Ketchum Mejía. 1999. Distribución geográfica y depredación de Porites lobata (Anthozoa: Scleractinia) en la costa occidental de México. Rev. Biol. Trop. 47: 273-279.

Reyes-Bonilla, H. \& L.E. Calderon-Aguilera. 1999. Population density, distribution and consumption rates of three corallivores at Cabo Pulmo Reef, Gulf of California, Mexico. PSNZI Mar. Ecol. 20: 347-357.

Reyes Bonilla, H., L.E. Calderon Aguilera, G. Cruz Piñon, P. Medina Rosas, R.A. López Pérez, M.D. Herrero Pérezrul, G.E. Leyte Morales, A.L. Cupul Magaña \& J.D. Carriquiry. 2005. Atlas de los corales pétreos (Anthozoa: Scleractinia) del Pacífico Mexicano. Centro de Investigación Científica y de Educación Superior de Ensenada, Comisión Nacional para el Conocimiento y Uso de la Biodiversidad, Consejo Nacional de Ciencia y Tecnología, Universidad de Guadalajara-Centro Universitario de la Costa, Universidad del Mar, Ensenada, Baja California, México.

Reyes-Bonilla, H., L.E. Calderón-Aguilera, G. Cruz-Piñón, R.A. López-Pérez \& P. Median-Rosas. 2010. Evaluación de la diversidad gama de corales arrecifales (Scleractinia) en el Pacífico de México. Rev. Mex. Biodiv. 81: 113-121.

Rodríguez-Troncoso, A.P., E. Carpizo-Ituarte, G.E. LeyteMorales, G. Chi-Barragán \& O. Tapia-Vázquez. 2011. Sexual reproduction of three coral species from the Mexican south Pacific. Mar. Biol. 158: 2673-2683.

Saavedra-Sotelo, N.C., L.E. Calderón-Aguilera, H. ReyesBonilla, R.A. López-Pérez, P. Medina-Rosas \& A. Rocha-Olivares. 2011. Limited genetic connectivity of Pavona gigantea in the Mexican Pacific. Coral Reefs 30: 677-686.

Salcedo-Martínez, S., G. Green, A. Gamboa-Contreras \& P. Gómez. 1988. Inventario de macroalgas y macroinvertebrados bénticos, presentes en áreas rocosas de la región de Zihuatanejo, Guerrero, México. An. Inst. Cienc. Mar. Limnol. UNAM 15: 73-96.

Sosa-Avalos, R., M.D. Martínez-Gaxiola \& G. GaxiolaCastro. 2006. Producción primaria en el Pacífico tropical mexicano estimada a partir de datos derivados de sensores remotos, p. 315-323. In M.C. JiménezQuiroz \& E. Espino-Barr (ed.). Los recursos pesqueros y acuícolas de Jalisco, Colima y Michoacán. Instituto Nacional de la Pesca, México.

Veron, J. 2000. Corals of the World. Australian Institute of Marine Science,Townsville, MC, Australia.

Zar, J.H. 2009. Biostatistical analysis. Prentice-Hall, San Francisco, EEUU. 